\title{
Development of a supported self-management intervention for adults with type 2 diabetes and a learning disability
}

Allan House ${ }^{1 *}$ (D) Gary Latchford ${ }^{1}$, Amy M. Russell ${ }^{1}$, Louise Bryant ${ }^{1}$, Judy Wright ${ }^{1}$, Elizabeth Graham², Alison Stansfield ${ }^{3}$, Ramzi Ajjan ${ }^{4}$ and On behalf of the OK Diabetes team

\begin{abstract}
Background: Although supported self-management is a well-recognised part of chronic disease management, it has not been routinely used as part of healthcare for adults with a learning disability. We developed an intervention for adults with a mild or moderate learning disability and type 2 diabetes, building on the principles of supported self-management with reasonable adjustments made for the target population.

Methods: In five steps, we:

1. Clarified the principles of supported self-management as reported in the published literature

2. Identified the barriers to effective self-management of type 2 diabetes in adults with a learning disability

3. Reviewed existing materials that aim to support self-management of diabetes for people with a learning disability

4. Synthesised the outputs from the first three phases and identified elements of supported self-management that were (a) most relevant to the needs of our target population and (b) most likely to be acceptable and useful to them

5. Implemented and field tested the intervention
\end{abstract}

Results: The final intervention had four standardised components: (1) establishing the participant's daily routines and lifestyle, (2) identifying supporters and their roles, (3) using this information to inform setting realistic goals and providing materials to the patient and supporter to help them be achieved and (4) monitoring progress against goals. Of 41 people randomised in a feasibility RCT, thirty five (85\%) completed the intervention sessions, with over three quarters of all participants (78\%) attending at least three sessions.

Twenty-three out of 40 (58\%) participants were deemed to be very engaged with the sessions and 12/40 (30\%) with the materials; 30 (73\%) participants had another person present with them during at least one of their sessions; 15/41 (37\%) were reported to have a very engaged main supporter, and 18/41 (44\%) had a different person who was not their main supporter but who was engaged in the intervention implementation.

Conclusions: The intervention was feasible to deliver and, as judged by participation and engagement, acceptable to participants and those who supported them.

Trial registration: Current Controlled Trials ISRCTN41897033 (registered 21/01/2013)

Keywords: Type 2 diabetes, Supported self-management, Learning disability

\footnotetext{
* Correspondence: a.o.house@leeds.ac.uk

${ }^{1}$ Leeds Institute of Health Sciences, University of Leeds, Worsley Building,

Leeds LS2 9NL, UK

Full list of author information is available at the end of the article
}

(c) The Author(s). 2018 Open Access This article is distributed under the terms of the Creative Commons Attribution 4.0 International License (http://creativecommons.org/licenses/by/4.0/), which permits unrestricted use, distribution, and reproduction in any medium, provided you give appropriate credit to the original author(s) and the source, provide a link to the Creative Commons license, and indicate if changes were made. The Creative Commons Public Domain Dedication waiver (http://creativecommons.org/publicdomain/zero/1.0/) applies to the data made available in this article, unless otherwise stated. 


\section{Background}

In UK practice, the terms learning disability and intellectually disability are used interchangeably. It is estimated that $2 \%$ of the population has a learning disability, with about $1.5 \%$ having a mild or moderate disability. Diabetes in people with a learning disability has been estimated to be more common than in the general population $[1,2]$, with a cited prevalence of $9-11 \%[3,4]$. People with learning disabilities also have higher rates of hospital admissions from diabetes-related ambulatory care-sensitive conditions [4]. There are a number of possible explanations for high rates of type 2 diabetes mellitus (T2DM) in adults with a learning disability including high prevalence of obesity $[5,6]$, prescription medications that increase diabetes risk and more limited selfmanagement skills [7].

Supported self-help or self-management with health problems is now well established in that the principles are clear in terms of core elements, although the intensity with which it is delivered and its specific content have varied considerably between studies [8-10]. In relation to intensity, the main variation is in amount of contact with the support/therapist, which ranges from regular face-to-face meetings to limited telephone contact. The usual pattern is that a professional (or trained peer) acts as a 'therapist' to help and encourage the nominated patient in using self-management materials.

As regards the content, all programmes contain an educational and an instructional component, the variation residing mainly in the degree of use of formal techniques for supporting change in behaviour and the degree to which they are theory-based. Typical elements [11] include:

- Helping people to understand the short-, medium- and longer term consequences of health-related behaviour

- Helping people to feel positive about the benefits of changing their behaviour

- Building the person's confidence in their ability to make and sustain changes

- Recognising how social contexts and relationships may affect a person's behaviour

- Helping plan changes in terms of easy steps over time

- Identifying and planning for situations that might undermine the changes people are trying to make (including planning explicit 'if-then' coping strategies to prevent relapse)

- Encouraging people to make a personal commitment to adopt health-enhancing behaviours by setting (and recording) achievable goals in particular contexts, over a specified time

- Helping people to use self-regulation techniques (such as self-monitoring, progress review, relapse management and goal revision) to encourage learning from experience
- Encouraging people to engage the support of others to help them to achieve their behaviour-change goals

Current recommendations for diabetes self-management focus largely on educational and didactic approaches [12]. Development of self-management material for adults with a learning disability has tended to take the same approach [13]. In these programmes, there is typically less emphasis on more autonomous aspects of self-management such as advice about self-monitoring. There is also little on the interaction between the person with diabetes and others supporting their care. Many adults with a learning disability do not live entirely independently even when living in the community - that is, not living in a hospital setting. Family members and other informal or formal carers often provide support in the form of help with shopping, cooking, monitoring and prompting about medication and so on. Living and support arrangements are diverse $[14,15]$; some adults with a learning disability remain in the parental home; some live with a sibling or other relative; some live alone or in shared accommodation with non-resident support or peer support from those with whom they share, and some are married or cohabiting with somebody who may or may not themselves have a learning disability. Since many of the positive and negative influences on good diabetes management reside in the immediate social network [16-20], selfmanagement needs to involve not just the person with diabetes but their supporter, and flexibility is needed in negotiating and implementing an intervention.

There is a clear need to improve on this state of affairs. The Equality Act 2010 sets out the legal requirement for public services to provide reasonable adjustments at both service level and individual level for people with a disability, and that should include provision of accessible therapeutic support. This will require modification of even well-established interventions since people with a learning disability have by definition a significantly reduced ability to understand new or complex information and manage independently [21]; many also have communication difficulties. It will involve finding a form of communication that matches the person's needs and may well involve a supporter who is familiar with helping that person make decisions $[22,23]$.

We have recently completed a feasibility RCT of a supported self-management intervention for evaluation in a feasibility RCT, the OK Diabetes trial. The protocol for the OK Diabetes RCT [24] and the results for the trial $[25,26]$ have been reported elsewhere. The present paper reports on our work in developing and field testing the intervention for use in the trial.

The approach we adopted in developing the intervention lies somewhere between the creation of a new 
therapy on the one hand and standardising (for example in a manual) an existing one. It involved using the principles of an existing therapy in developing the form and content of the intervention, with reasonable adjustments made so that it was suitable for use with our target population. This approach responds to the need to make reasonable adjustments to healthcare interventions for those with a learning disability [27] without sacrificing the effective components of a complex intervention that has been developed and evaluated in the general population.

\section{Methods}

Supported self-management is an approach to chronic disease management in which the underlying theory and the basic principles of implementation are already established. Development work in the current project did not therefore need to start from the early phases of intervention development (Fig. 1) as recommended by, for example, the UK's Medical Research Council [28]-elaborating theory and undertaking early phase proof of concept and efficacy studies. Instead we planned our development work in five phases:

1. Clarifying the principles of supported selfmanagement-as summarised in review articles and educational pieces and as identifiable from the protocols and final reports of individual studies of self-management in diabetes

2. Identifying from published literature the reported barriers to effective self-management of type 2 diabetes in adults with a learning disability
3. Collaborating with services that provide health support to adults with a learning disability to review existing materials that aim to support self-management of diabetes for people with a learning disability for examples of good practice

4. Synthesising the outputs from the first three phases to decide on those elements of supported self-management that are most relevant to the needs of our target population (that is, will overcome likely barriers) and most likely to be acceptable and useful (that is, match identified good practices).

This phase involved a series of problem structuring and consensus meetings in the research team, at each step checking interim outputs against guidelines on reasonable adjustments and consulting with experts and service users

5. Implementation and field testing of early versions-modifying the intervention materials in the light of feedback from research diabetes nurses supporting the intervention (see later)

At each stage, there were regular consultation meetings involving members of the research team, people with a learning disability and their representatives in local third sector organisations and expert clinicians experience working in learning disability.

We worked with two local third sector organisations with a special interest in supporting people with a learning disability: Tenfold (http://www.tenfold.org.uk/) and People in Action (http://peopleinaction.org.uk/) and with easy on the $i$, (www.easyonthei.nhs.uk) an organisation with special expertise in preparing accessible

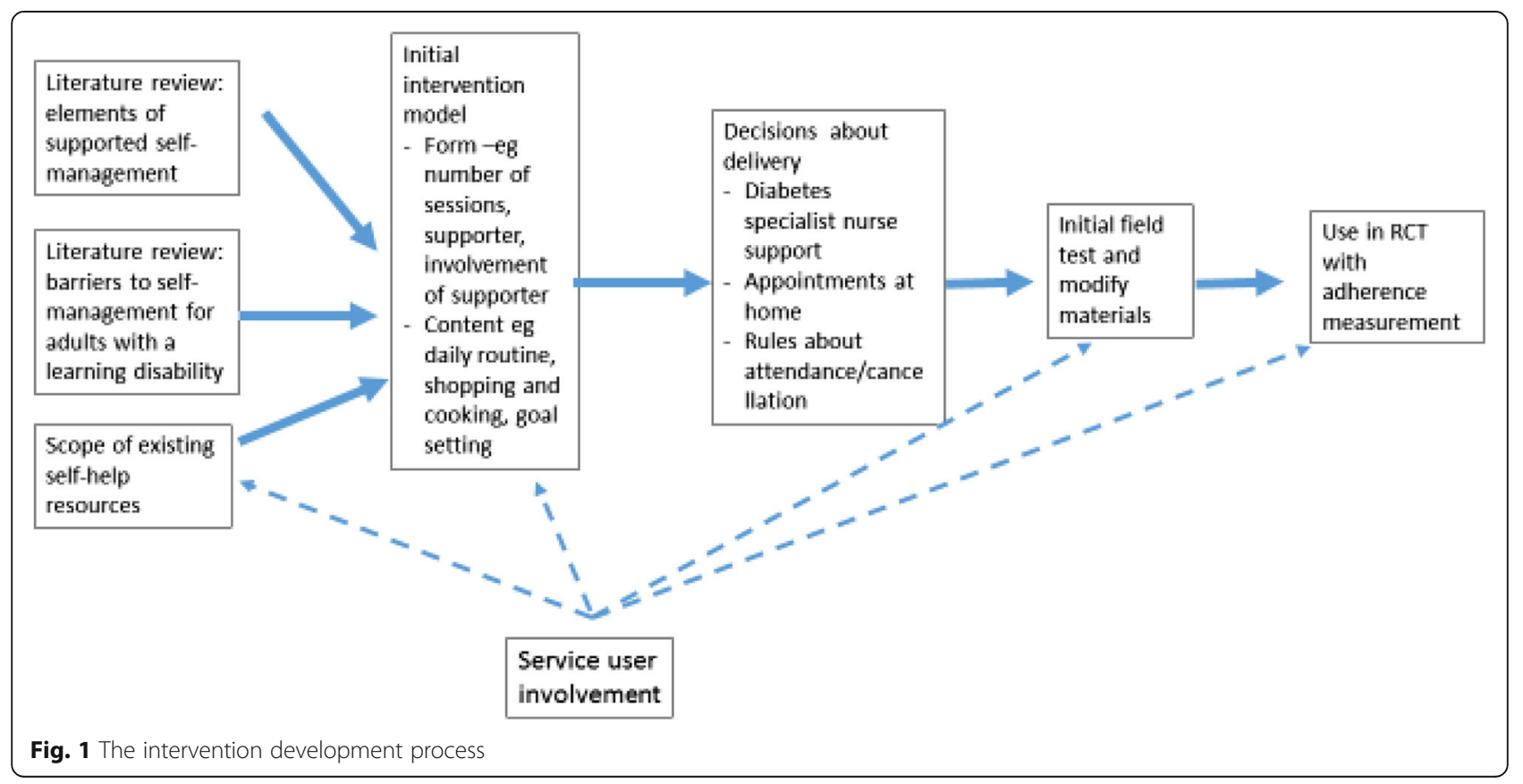


materials for people with a learning disability. Each group had established relationships with adults with a mild or moderate learning disability, with whom they worked to review materials and policies. Over 12 months, we were introduced to service users who could advise us, by the organisations for which they worked; we met two to three times for each part of the project. Exchanges were not formalised, and we did not record the meetings for research purposes, except to record the advice received.

\section{Phases $1+2$ : literature reviews}

At the time of our study, there were no relevant RCTs in the area of diabetes self-management in learning disability and therefore we did not undertake a formal systematic review of effectiveness studies.

We aimed to use the published literature to identify (a) the principles of self-management that we would hope to embody in our own intervention and (b) those influences on self-management potential in our population that should inform the form or content of a supported self-management programme.

To help identify published information relevant to our intervention, an information scientist (JW) undertook initial searches in four main areas:

- Supported self-management in chronic disease;

- Self-care in diabetes, including barriers to effective self-care;

- Diabetes and a learning disability-a broad search to identify factors that might be specific to the target population;

- Descriptions of specific interventions aimed at improving diabetes control in adults with a learning disability.

Searches were refined in a series of iterative discussions based upon outputs from preliminary searches. We also checked the reference lists of two relevant NICE Guidelines, [11, 29], two Cochrane Reviews, [30, 31 , a guideline on supported self-management published by Diabetes UK, [32], and outlines of national standards in diabetes management from the US [33] and the UK [34].

On the basis of these searches, run in MEDLINE, EMBASE, CINAHL and PsychINFO, we identified and reviewed 707 titles and abstracts on the topics of selfmanagement of chronic disease including diabetes, and 350 titles and abstracts on the topic of diabetes in adults with a learning disability.

The searches are available in the full report of the study [26].

Titles and abstracts were reviewed by two of the applicants (AH and GL), and full versions of relevant papers were obtained. We categorised retrieved papers as follows:

- Reviews of self-management and diabetes $(N=10)$ $[9,10,35-42]$

- Individual self-management programmes or interventions $(N=22)$ [43-64]

- Research protocols describing individual self-management interventions $(N=8)$ [65-72]

- Observational studies reporting influences on self-management-barriers and enablers $(N=31)$ [16-20, 73-99]

We extracted data that described the form or content of self-management using an initial framework derived from reviewing the two NICE Guidelines, two Cochrane Reviews and the guideline on supported selfmanagement published by Diabetes UK. We then refined this framework by reference to six individual studies for which reasonably comprehensive descriptions of the intervention were contained in the reports [51, 58, 100103]. The remaining studies were reviewed against this framework to identify any missing themes, using a modified best-fit framework analysis [104].

Next, we reviewed papers describing barriers to effective self-management, influences on interventions or outcomes that were specific to adults with a learning disability. In a series of review meetings, we organised the identified influences into a descriptive framework. We did not apply a quality assessment to papers, as long as they contained a usable account of intervention components.

Finally, we combined the two frameworks in a synthesis that allowed us to identify the general principles to be adhered to in implementing a pragmatic and sustainable programme of supported self-management, and the form and content of the specific intervention for this project.

\section{Phase 3: scoping existing resources}

For existing self-care resources, a scoping exercise was conducted in which examples of good practice in interventions around health in people with a learning disability were sought from services in the UK. Sources included charities including Diabetes UK, local NHS Trusts and patient groups, NHS Choices and Easyhealth.

We identified 18 examples of resources developed in the UK to support self-management of diabetes for people with a learning disability. The resources were all in leaflet or booklet form, using more-or-less easy-read language, illustrated with photographs or cartoons and covering topics like foods to eat and avoid, exercise, foot care and what to do if ill.

Resources were reviewed by a panel consisting of members of the research team, a dietician in learning 
disability services and service user representatives. Panel members rated each resource from 1 to 10 on a Likert scale. In informal group discussions that reviewed each resource in turn, members identified which aspects they liked and disliked about each resource (for example, information complexity, font size, use of images) and noted any implications for the new intervention. Results were recorded on a structured proforma.

\section{Phase 4: problem structuring and priority setting}

Using the general approach of problem structuring and priority setting [105], preliminary versions of the supported self-management package-including not just format and content but tailoring (for easy reading, visibility for those with poor acuity and so on)-were discussed in the research team. Finally, we considered guidance on reasonable adjustments to healthcare designed to ensure access for people with a disability, to check that we were meeting these obligations.

A further round of more focused (purposive) reviewing of literature was used to clarify which were the key principles for this population. We generated two checklists derived from the literature and our discussions (see Tables 1 and 2 below) to ensure that all relevant topics were covered, that all components of the intervention could be linked to principles of self-management, and to help frame final decisions (Table 3).

Principles for priority setting were:

- The intervention should respond to known barriers to self-management reported by people with a disability-including practical problems such as transport, likely attrition from drop out when multiple attendances are expected, inability to accommodate the presence of a supporter;

- The format of the intervention should be likely to encourage self-maintained change beyond an early supported element; in our target population, this meant especially that the intervention should involve supporters involved with any aspect of lifestyle (shopping, food choice, physical activity, medication monitoring and so on) relevant to diabetes;

- The intervention should be designed to be readily integrated into usual healthcare provision in the NHS, to ensure sustainability.

Based upon all the advice we received from those working with our target group, we wanted to give particular salience to:

- Practical aspects of self-care-buying and preparing food. changes aiming for a healthier balanced diet, increasing physical activity
Table 1 Elements of supported self-management for type 2 diabetes

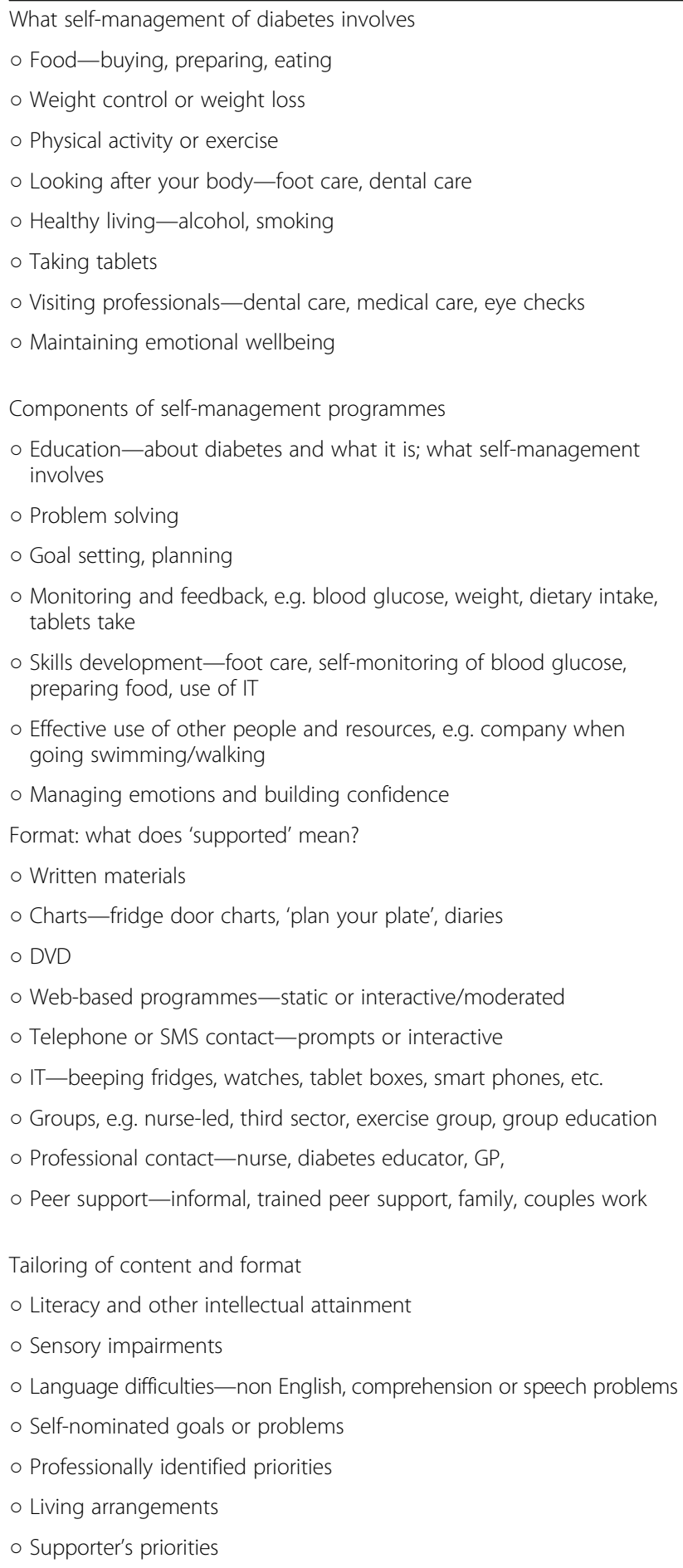

- Use of simple (accessible) written materials and charts

- Supportive contact both with a professional and with a supporter if one could be identified

- Use of practical goal setting, planning to meet goals and self-monitoring 
Table 2 Checklist of possible needs and barriers to good healthcare requiring adjustment

\begin{tabular}{|c|c|}
\hline Example of impairment or deficit & Example of adjustment (enabler of good healthcare) \\
\hline Intellectual disability/reduced mental capacity & Staff training in capacity assessment and inclusive practice \\
\hline Memory problems & Prompts, support for appointments \\
\hline Literacy/reading skills deficit & Accessible materials, communication skills \\
\hline Vision/hearing impairment & Visual aids \\
\hline Speech problems & Time, trained staff \\
\hline Mobility difficulties, physical symptoms or restrictions & May need OT/physio assessment/mobility aids \\
\hline \multicolumn{2}{|l|}{ Attitudinal barriers } \\
\hline History of lack of dignity/respect in services & Staff training \\
\hline Threat to safety including bullying & Safeguarding protocols \\
\hline Overcoming stigma & Advocacy \\
\hline \multicolumn{2}{|l|}{ Instrumental barriers } \\
\hline Transport to services & Funding, safe provision \\
\hline Finance & Personal budget \\
\hline Lack of access to personal pleasure/R+R activities & Planning meeting with supporter \\
\hline Treatment burden—timing, side-effects & Support with adherence, modified regime \\
\hline \multicolumn{2}{|l|}{ Social barriers } \\
\hline Lack of social support/networks & Identify, train and support carers; advocacy; third sector \\
\hline Talking with professionals & Staff training + supervision \\
\hline Communicating needs & LD register; Health Action Plan \\
\hline Understanding — health risks, necessary actions & Accessible information \\
\hline Low self confidence & Social engagement activities \\
\hline \multicolumn{2}{|l|}{ Mental Health } \\
\hline Challenging behaviour & Pacing of change; staff training \\
\hline Distress + mental disorder & Mental health review with learning disability team \\
\hline
\end{tabular}

By contrast, we decided that less helpful aspects would be:

- Education of a more theoretical sort about the nature of diabetes, food values and so on.

All participants received factual information about managing their diabetes in a booklet as part of the Treatment As Usual arm of the RCT (https://www.diabetes.org.uk/about_us/news/ learning-disabilities-leaflet)

- IT-based interventions, web, DVD, mobile phone etc., because this is usually not readily accessible by our participants

- Group-based interventions-attendance is typically poor and meeting the specific individual needs arising in a heterogeneous population is harder

Phase 5: implementation and field testing an early version We decided that professional support would be provided by diabetes nurses with experience in primary care rather than learning disability nurses, the rationale being that (i) very few of the target population would be in contact with (or taken on by) specialist learning disability services; (ii) in routine NHS practice where $20-25 \%$ of diabetes patients would be using insulin, diabetes nurses would have more relevant experience; (iii) for our population (not all of whom would have been told they had, or would self-describe as having, a learning disability), the diabetes background was considered to be more acceptable; and (iv) the intervention would be more readily easily used by staff in mainstream services. We planned for the sessions to be delivered in people's homes to ensure adherence and to allow assessment of the person's everyday environment, since we did not think the participants would be able to give a good account in a clinic of the influences on their diet, physical activity and self-care.

We developed a training plan for the nurses delivering the intervention covering the underlying principles of mental capacity and of self-management, the individualised elements specific to learning disability, trouble- 
Table 3 Links between components of OK Diabetes intervention and principles of self-management

\begin{tabular}{lr}
\hline Behaviour change: the principles of effective interventions [29] \\
\hline Principle & Place in intervention programme \\
\hline
\end{tabular}

Helping people to understand the short-, medium- and longer term consequences of health-related behaviour

Helping people to feel positive about the benefits of changing their behaviour

Building the person's confidence in their ability to make and sustain changes

Recognising how social contexts and relationships may affect a person's behaviour

Helping plan changes in terms of easy steps over time

Identifying and planning for situations that might undermine the changes people are trying to make (including planning explicit 'if-then' coping strategies to prevent relapse)

Encouraging people to make a personal commitment to adopt health-enhancing behaviours by setting (and recording) achievable goals in particular contexts, over a specified time

Helping people to use self-regulation Identify personal rewards for success techniques (such as self-monitoring, progress review, relapse

management and goal revision)

to encourage learning from

experience

Encouraging people to engage the support of others to help them to

maintain their behaviour-change

goals

shooting and dealing with potential problems and the details of the programme and the materials provided with it.

Explicit links were made between the practicalities of the intervention and its rationale in self-management principles (Table 3). We interviewed two general practitioners, a consultant physician in diabetes and a diabetes nurse manager, about any challenges in fitting the intervention into routine care.

The training programme was delivered by two of the researchers $(\mathrm{AH}, \mathrm{GL})$ over three sessions of face-to-face contact with the nurses. Both trainers were clinicians (liaison psychiatrist, $\mathrm{AH}$, clinical psychologist GL) with experience of NHS practice in the management of longterm physical illness and previous experience in applied health research and supervision of therapies. We did not involve service users in the training. An additional session on mental capacity assessment was delivered by AS to the nurses and all research interviewers.

Supervised use of the intervention with three initial cases was also arranged. In each case, the whole intervention was delivered over a maximum of four visits and the nurses met together with $\mathrm{AH}$ and GL after each visit, to discuss any challenges with implementation. On the basis of this experience, early versions of the intervention were modified in format to make them easier to use. In particular, the forms for keeping notes on each contact were found to be over-structured by the nurses and intrusive for use in the field. Once they had familiarised themselves with the principles of selfmanagement and the nature of each contact, the nurses preferred to make free-form notes during contact and then check afterwards that they had recorded all the necessary information and completed the essential standardised forms (CRF) for the trial.

Both nurses involved in the field-testing reported during case supervision finding the materials easy to use and the nature of the intervention easy to understand.

\section{Results (see Additional files 1, 2 and 3)}

The final intervention had four standardised components with associated materials. How they were delivered depended on participant and supporter characteristics and preferences:

1. Establishing the participant's daily routines and lifestyle: This included current diet and activity routines, participation in daytime social activities or work, shopping and food preparation, current self-reported health and self-management. The main aim of this component was to identify the social and personal influences in the life of the person with diabetes that would limit their ability to selfmanage or that might be mobilised as a resource in supporting self-management.

2. Identifying all supporters and helpers and their roles: A key supporter and other helpers were identified where possible. Key supporters and other helpers were given written information about the project, and if they agreed to support a goal set by the participant, they were given a written reminder of their role. The main aim was to identify people who might be a useful resource in supporting self-management and to ensure any changes were embedded in the social network for longer term maintenance of change.

3. Setting realistic goals for change: The main aim was to avoid prescribing change in the way of good dietary practice or other lifestyle change, but to support goals suggested by the person with diabetes 
that were specific, simple and achievable given the person's current routines and social support, and consonant with their willingness to make change. The intention was to encourage engagement in a population usually thought of as having little agency and to introduce the idea of selectable elements in a repertoire of self-management options.

4. Monitoring progress against agreed upon goals: We devised a simple system that did not depend on high levels of functional literacy, using tear-off calendar sheets on which participants noted goal attainment in a Yes/No format. The main aim was to encourage active participation in an activity that is a core feature of self-management.

We prepared materials to accompany these activities:

- For the nurses-templates for a weekly timetable, a chart to record friends and family and other helpers, charts to be completed in collaboration with the person with diabetes ('my life', 'my likes and don't likes', 'looking after my diabetes')

- For the person with diabetes-an OK Diabetes board to place in a prominent position at home with visible record of goals including pictorial prompts, e.g. 'snack swaps', a written action plan in multiple formats and tear-off slips to record daily actions

- For supporters and helpers-an information sheet explaining the study and a card summarising what their role was in helping to support the person with diabetes in meeting their chosen goals

The research nurse worked through the elements of supported self-management with the participant, explaining how to use materials and suggesting initial actions and activities. Further contact was negotiated with the person with diabetes. We anticipated that a total of three to four meetings of 30 to 60 min over 6 to 8 weeks would be provided, followed by telephone support and advice.

We took steps to ensure consistency in the use of the supported self-management: (i) training and supervision sessions with research nurses, (ii) annotation of the intervention materials by research nurses and (iii) ensuring nurses had other experience and training in diabetes or learning disability care prior to the RCT.

\section{Results}

In developing the intervention, we were mindful of the need to assess fidelity (how much the intervention was delivered as planned) and adherence (how successfully the intervention was taken up by participants). We developed a simple approach to collecting intervention materials that could then be used to assess these aspects of intervention performance, the details of which have been reported elsewhere $[25,106]$.

\section{Discussion}

Although not described in the same detail, similar approaches have been used previously in adapting established and effective therapies to develop brief psychological interventions for depression in people with a learning disability $[107,108]$.

Our approach to intervention development was based upon the principle of making reasonable adjustments to an existing approach and has a number of advantages. Firstly, because it involved considerable service user and expert input, it proved acceptable to participants. Secondly, its flexibility, which includes when, where and by whom is it delivered, makes our findings transferable to other settings.

As others have noted however [109], even extensively documented and theory-driven approaches to intervention development will entail a degree of personal judgement and will depend upon the particular perspective of the service users and third sector organisations consulted. We are aware for example, that while we privileged an understanding of the individual's lifestyle and relationship with supporters over psychological characteristics like motivation or knowledge, others have chosen to adapt existing educational programmes for use with this population [110].

\section{Conclusions}

Existing evidence-based interventions can be successfully modified for use with adults who have a learning disability, using literature reviews, service user and expert input to decide upon principle focus and upon desirable form and content of the eventual package. Services can be delivered by healthcare staff with limited experience of LD with relatively little training. Flexibility will always be needed to respond to the differing living and personal arrangements of participants (for example the nature and involvement of a supporter) and variable cognitive abilities.

\section{Additional files}

Additional file 1: Adherence to intervention checklist. (DOCX $178 \mathrm{~kb}$ )

Additional file 2: How to sheet: Snack swaps. How to: eat more fruit. How to: be more active. How to: eat more vegetables. (DOCX 7684 kb)

Additional file 3: Intervention materials. (DOCX 209 kb)

\section{Acknowledgements}

Other members of the OK Diabetes team were as follows: Professor Amanda Farrin, Dr. Alexandra Wright-Hughes, and Dr. Rebecca Walwyn (Leeds Clinical Trial Research Unit); Professor Claire Hulme and Mr. John O'Dwyer (Academic Unit of Health Economics). 
We received valuable support and input from two local third sector organisations and would like to acknowledge especially Kath Lindley at Tenfold http://www.tenfold.org.uk/ and Aqila Choudhury at People in Action http://peopleinaction.org.uk/. 'easy on the $\mathrm{i}$ ' is the information design service within the Learning Disability Service at Leeds and York Partnership NHS Foundation Trust http://www.easyonthei.nhs.uK/. They contributed greatly at each stage of development and in generating the materials to support the intervention.

\section{Funding}

The study was funded by the National Institute for Health Research Health Technology Assessment programme NIHR HTA project 10/102/03.

\section{Availability of data and materials}

The dataset used during the current study will be made available to the scientific community with as few restrictions as feasible while retaining exclusive use until the publication of major outputs. Enquiries should be directed to the corresponding author.

\section{Authors' contributions}

$\mathrm{AH}$ contributed to the design analysis and write up of the project and was the chief investigator overseeing the project implementation and corresponding author for the report. LB contributed to the design, conduct and analysis of the report, with lead role on the research in adults with learning disability, deputised for Professor House. AM was the project co-ordinator, responsible for the design of materials, recruitment and data collection, and JW designed and undertook all literature searches. GL contributed to the design and implementation of the SSM intervention, including supervision of nurses. AS lead the role in advising on involving adults with learning disability, training researchers in practice related to mental capacity. RA advised on all clinical aspects of the treatment and assessment of type 2 diabetes. All authors read and approved the final manuscript.

\section{Ethics approval and consent to participate}

Ethical approval was granted for the study by the Yorkshire and Humber Research Ethics Committee (Reference: 12/ YH/0304). All participants gave written or independently witnessed verbal consent.

\section{Competing interests}

The authors declare that they have no competing interests.

\section{Publisher's Note}

Springer Nature remains neutral with regard to jurisdictional claims in published maps and institutional affiliations.

\section{Author details}

${ }^{1}$ Leeds Institute of Health Sciences, University of Leeds, Worsley Building, Leeds LS2 9NL, UK. '2Leeds Institute of Clinical Trials Research, University of Leeds, Worsley Building, Leeds LS2 9NL, UK. ${ }^{3}$ Leeds York Partnership NHS Foundation Trust, Leeds, UK. ${ }^{4}$ Division of Cardiovascular and Diabetes Research, University of Leeds, Leeds, UK.

\section{Received: 3 November 2017 Accepted: 9 May 2018}

\section{Published online: 29 May 2018}

\section{References}

1. Straetmans JM, van Schrojenstein Lantman-de HM, Schellevis FG, Dinant GJ. Health problems of people with intellectual disabilities: the impact for general practice. Br J Gen Pract. 2007;57(534):64-6.

2. Emerson E, Baines S, Allerton L, Welch V. Health inequalities and people with learning disabilities in the UK: 2010. Durham: Improving Health \& Lives: Learning Disabilities Observatory. p. 2010.

3. Emerson E. Health status and health risks of the 'hidden majority' of adults with intellectual disability. Intellect Dev Disabil. 2011;49

4. Glover G, Emerson E, Eccles R: Using local data to monitor the health needs of people with learning disabilities. Durham: Lives: Learning Disabilities Public Health Observatory; 2012.

5. Hsieh K, Rimmer JH, Heller T. Obesity and associated factors in adults with intellectual disability. J Intellect Disabil Res. 2014;58(9):851-63.

6. Melville C, Hamilton S, Hankey C, Miller S, Boyle S. The prevalence and determinants of obesity in adults with intellectual disabilities. Obes Rev. 2007;8
7. Hert M, Correll CU, Bobes J, Cetkovick-Bakmas M, Cohen D, Asai I, Detraux J, Gautam S, Moller HJ, Ndetei DM. Physical illness in patients with severe mental disorders. I. Prevalence, impact of medications and disparities in health care. World Psychiatry. 2011;10(1):52-77.

8. Newman S, Steed L, Mulligan K. Self-management interventions for chronic illness. Lancet. 2004:364(9444):1523-37.

9. Heinrich E, Schaper NC, de Vries NK. Self-management interventions for type 2 diabetes: a systematic review. Eur Diabetes Nurs. 2010;7(2):71-6.

10. Radhakrishnan K. The efficacy of tailored interventions for self-management outcomes of type 2 diabetes, hypertension or heart disease: a systematic review. J Adv Nurs. 2012;68(3):496-510.

11. Type 2 diabetes: prevention in people at high risk [https://www.nice.org.uk/ guidance/ph38]. Accessed 21 May 2018

12. National Institute for Health and Clinical Excellence: Type 2 diabetes in adults: management. NICE guideline NG28. In.: National Institute for Health and Care Excellence; 2015.

13. Taggart L, Coates V, Clarke M, Truesdale-Kennedy M, Bunting B. Piloting a structured education programme for the self-management of type 2 diabetes for adults with intellectual disabilities: study protocol for a pilot randomised controlled trial. Trials. 2015;16(148):1-8.

14. Braddock D, Emerson E, Felce D, Stancliffe RJ. Living circumstances of children and adults with mental retardation or developmental disabilities in the United States, Canada, England and Wales, and Australia. Dev Disabil Res Rev. 2001;7(2):115-21.

15. Forrester-Jones R, Carpenter J, Coolen-Schrijner P, Cambridge P, Tate A, Beecham J, Hallam A, Knapp M, Wooff D. The social networks of people with intellectual disability living in the community 12 years after resettlement from long-stay hospitals. J Appl Res Intellect Disabil. 2006;19(4):285-95.

16. Cardol M, Rijken M, van Schrojenstein Lantman-de Valk H. Attitudes and dilemmas of caregivers supporting people with intellectual disabilities who have diabetes. Patient Educ Couns. 2012;87(3):383-8.

17. Hale LA, Trip HT, Whitehead L, Conder J. Self-management abilities of diabetes in people with an intellectual disability living in New Zealand. J Policy Pract Intellect Disabil. 2011;8(4):223-30.

18. Rintala T-M, Jaatinen P, Paavilainen E, Åstedt-Kurki P. Interrelation between adult persons with diabetes and their family a systematic review of the literature. J Fam Nurs. 2013;19(1):3-28.

19. Stopford R, Winkley K, Ismail K. Social support and glycemic control in type 2 diabetes: a systematic review of observational studies. Patient Educ Couns. 2013:93(3):549-58.

20. Chesla CA, Bartz RJ, Gilliss C, Skaff MA, Sabogal F, Kanter RA, Lutz CP. The family and type 2 diabetes: a framework for intervention. Diabetes Educ. 1998;24(5):599-607.

21. Department of Health. Valuing people: a new strategy for learning disability for the 21st century. London: HM Government; 2001.

22. lacono T. Ethical challenges and complexities of including people with intellectual disability as participants in research. J Intellect Dev Disabil. 2006;31(3):173-9.

23. lacono T, Murray V. Issues of informed consent in conducting medical research involving people with intellectual disability. J Appl Res Intellect Disabil. 2003;16(1):41-51

24. Walwyn RE, Russell AM, Bryant LD, Farrin AJ, Wright-Hughes AM, Graham EH, Hulme C, O'Dwyer JL, Latchford GJ, Stansfield AJ. Supported self-management for adults with type 2 diabetes and a learning disability (OK-Diabetes): study protocol for a randomised controlled feasibility trial. Trials. 2015;16(1):342.

25. House A, Bryant L, Russell AM, Wright-Hughes A, Graham L, Walwyn R, Wright JM, Hulme C, O'Dwyer JL, Latchford G, et al. Randomized controlled feasibility trial of supported self-management in adults with type 2 diabetes mellitus and an intellectual disability: OK Diabetes. Diabet Med. 2018;35(6): 776-88.

26. House A, Bryant L, Russell AM, Wright-Hughes AM, Graham EH, Walwyn R, Wright J, Hulme CT, O'Dwyer J, Latchford GJ et al. Managing with learning disability and diabetes-OK-DIABETES: a case finding study and feasibility randomised controlled trial. In: NIHR Library. Edited by NIHR. London; 2018. in press

27. Equality Act, 2010. HM Government Equalities Office. https://www.gov.uk/ guidance/equality-act-2010-guidance.

28. Craig P, Dieppe P, Macintyre S, Michie S, Nazareth I, Petticrew M. Developing and evaluating complex interventions: the new Medical Research Council guidance. BMJ. 2008;337:a1655.

29. National Institute of Health and Care Excellence: Behaviour change: general approaches. Public Health Guideline PH6 2007. 
30. Deakin T, McShane CE, Cade JE, Williams RD. Group based training for selfmanagement strategies in people with type 2 diabetes mellitus. Cochrane Database Syst Rev. 2005;18

31. Duke SA, Colagiuri S, Colagiuri R. Individual patient education for people with type 2 diabetes mellitus. Cochrane Database Syst Rev. 2009;(1): CD005268.

32. Improving supported self-management for people with diabetes [https:// www.diabetes.org.uk/Documents/Reports/Supported_self-management.pdf]. Accessed 21 May 2018.

33. Funnell MM, Brown TL, Childs BP, Haas LB, Hosey GM, Jensen B, Maryniuk M, Peyrot M, Piette JD, Reader D, Siminerio LM. National standards for diabetes self-management education. Diabetes Care. 2009;32(Supplement 1):S87-94.

34. National Diabetes Audit - 2013-2014 and 2014-2015: Report 1, Care Processes and Treatment Targets. [http://www.hscic.gov.uk/catalogue/ PUB19900]. Accessed 21 May 2018.

35. Chrvala CA, Sherr D, Lipman RD. Diabetes self-management education for adults with type 2 diabetes mellitus: a systematic review of the effect on glycemic control. Patient Education. 2016;99(6):26-43.

36. Clark M. Diabetes self-management education: a review of published studies. Primary Care Diabetes. 2008;2(3):113-20.

37. Gucciardi E, Chan WW-S, Manuel L, Sidani S. A systematic literature review of diabetes self-management education features to improve diabetes education in women of Black African/Caribbean and Hispanic/Latin American ethnicity. Patient Educ Couns. 2013;92(2):235-45.

38. Ismail K, Winkley K, Rabe-Hesketh S. Systematic review and metaanalysis of randomised controlled trials of psychological interventions to improve glycaemic control in patients with type 2 diabetes. Lancet. 2004;363(9421):1589-97.

39. Jarvis J, Skinner T, Carey M, Davies M. How can structured self-management patient education improve outcomes in people with type 2 diabetes? Diabetes Obes Metab. 2010;12(1):12-9.

40. Norris SL, Lau J, Smith SJ, Schmid CH, Engelgau MM. Self-management education for adults with type 2 diabetes: a meta-analysis of the effect on glycemic control. Diabetes Care. 2002;25(7):1159-71.

41. Shrivastava SR, Shrivastava PS, Ramasamy J. Role of self-care in management of diabetes mellitus. J Diabetes Metab Disord. 2013;12(1):14

42. Steinsbekk A, Rygg L, Lisulo M, Rise MB, Fretheim A. Group based diabetes self-management education compared to routine treatment for people with type 2 diabetes mellitus. A systematic review with meta-analysis. BMC Health Serv Res. 2012;12(1):213.

43. Training in flexible, intensive insulin management to enable dietary freedom in people with type 1 diabetes: dose adjustment for normal eating (DAFNE) randomised controlled trial. British medical Journal. 2002;325:746-9.

44. Deakin TA, Cade JE, Williams R, Greenwood DC. Structured patient education: the diabetes X-PERT Programme makes a difference. Diabet Med. 2006;8

45. Glasgow R, Toobert D, Hampson S. Effects of a brief office-based intervention to facilitate diabetes dietary self-management. Diabetes Care. 1996;19(8):835-42.

46. Jansink R, Braspenning J, Keizer E, van der Weijden T, Elwyn G, Grol R. No identifiable $\mathrm{Hb} 1 \mathrm{Ac}$ or lifestyle change after a comprehensive diabetes programme including motivational interviewing: a cluster randomised trial. Scand J Prim Health Care. 2013;31(2):119-27.

47. Khunti K, Gray LJ, Skinner T, Carey ME, Realf K, Dallosso H, Fisher H, Campbell M, Heller S, Davies MJ. Effectiveness of a diabetes education and self management programme (DESMOND) for people with newly diagnosed type 2 diabetes mellitus: three year follow-up of a cluster randomised controlled trial in primary care. BMJ. 2012;344

48. Klug C, Toobert DJ, Fogerty M. Healthy changes for living with diabetes: an evidence-based community diabetes self-management program. Diabetes Educ. 2008;34(6):1053-61.

49. Lennox N, Edie G, Taylor M, Rey-Conde T, McPhee J. Diabetes, to the point: designing a website about diabetes for adults with intellectual disability and carers. Technol Disabil. 2009;21(1-2):11-8.

50. Lennox NG, Rey-Conde TF, McPhee JH, Taylor M. The development and evaluation of resources about diabetes management for people with intellectual disability and their care providers. J Dev Disabil. 2009;15(2):77-80.

51. Liu S, Bi A, Fu D, Fu H, Luo W, Ma X, Zhuang L. Effectiveness of using group visit model to support diabetes patient self-management in rural communities of Shanghai: a randomized controlled trial. BMC Public Health. 2012;12(1):1043.
52. Lorig K, Sobel D, Stewart A, Brown BW, Bandura A, Ritter P, Gonzalez VM, Laurent DD, Holman HR. Evidence suggesting that a chronic disease selfmanagement program can improve health status while reducing hospitalization: a randomized trial. Med Care. 1999;37

53. McGowan PT. Self-management education and support in chronic disease management. Primary care. 2012;39(2):307-25.

54. McMahon GT, Fonda SJ, Gomes HE, Alexis G, Conlin PR. A randomized comparison of online- and telephone-based care management with internet training alone in adult patients with poorly controlled type 2 diabetes. Diabetes Technol Ther. 2012;14(11):1060-7.

55. Mitchell B, Armour C, Lee M, Song YJ, Stewart K, Peterson G, Hughes J, Smith L, Krass I. Diabetes Medication Assistance Service: the pharmacist's role in supporting patient self-management of type 2 diabetes (T2DM) in Australia. Patient Educ Couns. 2011;83(3):288-94.

56. Molsted S, Tribler J, Poulsen PB, Snorgaard O. The effects and costs of a groupbased education programme for self-management of patients with type 2 diabetes. A community-based study. Health Educ Res. 2012:27(5):804-13.

57. Müller N, Stengel D, Kloos C, Ristow M, Wolf G, Müller UA. Improvement of $\mathrm{HbA}(1 \mathrm{c})$ and stable weight loss 2 years after an outpatient treatment and teaching program for patients with type 2 diabetes without insulin therapy based on urine glucose self-monitoring. Int J Gen Med. 2012;5:241-7.

58. Naik AD, Palmer N, Petersen NJ, Street RL Jr, Rao R, Suarez-Almazor M, Haidet P. Comparative effectiveness of goal setting in diabetes mellitus group clinics: randomized clinical trial. Arch Intern Med. 2011;171(5):453-9.

59. Song MS, Kim HS. Intensive management program to improve glycosylated hemoglobin levels and adherence to diet in patients with type 2 diabetes. Appl Nurs Res. 2009;22(1):42-7.

60. Thoolen BJ, de Ridder D, Bensing J, Gorter K, Rutten G. Beyond good intentions: the role of proactive coping in achieving sustained behavioural change in the context of diabetes management. Psychol Health. 2009;24(3):237-54.

61. Trento M, Passera P, Borgo E, Tomalino M, Bajardi M, Cavallo F, Porta M. A 5year randomized controlled study of learning, problem solving ability, and quality of life modifications in people with type 2 diabetes managed by group care. Diabetes Care. 2004;27(3):670-5.

62. van der Wulp I, de Leeuw JR, Gorter KJ, Rutten GE. Effectiveness of peer-led self-management coaching for patients recently diagnosed with type 2 diabetes mellitus in primary care: a randomized controlled trial. Diabet Med. 2012;29(10):e390-7.

63. Zanetti ML, Otero LM, Peres DS, MAd S, FPdM G, MCF F. Progress of the patients with diabetes mellitus who were managed with the staged diabetes management framework. Acta Paulista de Enfermagem. 2007;20:338-44.

64. Diabetes Prevention Program Research Group. Reduction in the incidence of type 2 diabetes with lifestyle intervention or metformin. N Engl J Med. 2002;2002(346):393-403

65. Christie D, Strange V, Allen E, Oliver S, Wong ICK, Smith F, Cairns J, Thompson R, Hindmarsh P, O'Neill S, et al. Maximising engagement, motivation and long term change in a Structured Intensive Education Programme in Diabetes for children, young people and their families: Child and Adolescent Structured Competencies Approach to Diabetes Education (CASCADE). BMC Pediatr. 2009;9(1):57.

66. Debussche X, Collin F, Fianu A, Balcou-Debussche M, Fouet-Rosiers I, Koleck $M$, Favier F. Structured self-management education maintained over two years in insufficiently controlled type 2 diabetes patients: the ERMIES randomised trial in Reunion Island. Cardiovasc Diabetol. 2012;11:91.

67. Gray LJ, Khunti K, Williams S, Goldby S, Troughton J, Yates T, Gray A, Davies MJ. Let's prevent diabetes: study protocol for a cluster randomised controlled trial of an educational intervention in a multi-ethnic UK population with screen detected impaired glucose regulation. Cardiovasc Diabetol. 2012;11(1):56

68. Johnson ST, Mundt C, Soprovich A, Wozniak L, Plotnikoff RC, Johnson JA. Healthy eating and active living for diabetes in primary care networks (HEALD-PCN): rationale, design, and evaluation of a pragmatic controlled trial for adults with type 2 diabetes. BMC Public Health. 2012;12:455.

69. Lascar N, Kennedy A, Jackson N, Daley A, Dowswell G, Thompson D, Stokes K, Greenfield S, Holder R, Andrews R et al: Exercise to preserve beta cell function in recent-onset type 1 diabetes mellitus (EXTOD) - a study protocol for a pilot randomized controlled trial. Trials 2013, 14:180-180.

70. Ma J, King AC, Wilson SR, Xiao L, Stafford RS. Evaluation of lifestyle interventions to treat elevated cardiometabolic risk in primary care (E-LITE): a randomized controlled trial. BMC Fam Pract. 2009;10:71. 
71. Murray E, Dack C, Barnard M, Farmer A, Li J, Michie S, Pal K, Parrott S, Ross J, Sweeting M. HeLP-Diabetes: randomised controlled trial protocol. BMC Health Serv Res. 2015;15(1):578.

72. Simmons D, Cohn S, Bunn C, Birch K, Donald S, Paddison C, Ward C, Robins P, Prevost AT, Graffy J. Testing a peer support intervention for people with type 2 diabetes: a pilot for a randomised controlled trial. BMC family practice. 2013;14(1):5. https://doi.org/10.1186/1471-2296-14-5.

73. Baker RM. Economic rationality and health and lifestyle choices for people with diabetes. Soc Sci Med. 2006;63(9):2341-53.

74. Bayliss EA, Steiner JF, Fernald DH, Crane LA, Main DS. Descriptions of barriers to self-care by persons with comorbid chronic diseases. Ann Fam Med. 2003;1(1):15-21.

75. Beverly EA, Miller CK, Wray LA. Spousal support and food-related behavior change in middle-aged and older adults living with type 2 diabetes. Health Educ Behav. 2007;

76. Cardol M, Rijken M, van Schrojenstein Lantman-de Valk H. People with mild to moderate intellectual disability talking about their diabetes and how they manage. J Intellect Disabil Res. 2012;56(4):351-60.

77. Cartwright L, Reid M, Hammersley R, Blackburn C, Glover L. Food choice by people with intellectual disabilities at day centres: a qualitative study. J Intellect Disabil. 2014;19(2):103-15.

78. Caton S, Chadwick D, Chapman M, Turnbull S, Mitchell D, Stansfield J. Healthy lifestyles for adults with intellectual disability: knowledge, barriers, and facilitators. J Intellect Dev Disabil. 2012;37(3):248-59.

79. Chlebowy DO, Hood S, LaJoie AS. Facilitators and barriers to selfmanagement of type 2 diabetes among urban African American adults focus group findings. Diabetes Educ. 2010;36(6):897-905.

80. Dagnan D. Psychosocial interventions for people with intellectual disabilities and mental ill-health. Curr Opin Psychiatry. 2007;20(5):456-60.

81. Ding EL, Prescott MR, Watson KT, Bui N, Makarechi L, Zoughbie DE: Abstract 009: Microclinic social network lifestyle intervention for weight loss and obesity management: a 10-month randomized controlled trial. Circulation 2013, 127(Suppl 12):A009-A009.

82. Dysch C, Chung MC, Fox J. How do people with intellectual disabilities and diabetes experience and perceive their illness? J Appl Res Intellect Disabil. 2012;25(1):39-49.

83. Gill JM, Mainous AG, Diamond JJ, Lenhard MJ. Impact of provider continuity on quality of care for persons with diabetes mellitus. Ann Fam Med. 2003; 1(3):162-70.

84. Glasgow R, Nelson C, Strycker L, King D. Using RE-AlM metrics to evaluate diabetes self-management support interventions. Am J Prev Med. 2006; 30(1):67-73.

85. Glasgow RE, Toobert DJ, Gillette CD. Psychosocial barriers to diabetes selfmanagement and quality of life. Diabetes Spectrum. 2001;14(1):33-41.

86. Gomersall T, Madill A, Summers LKM. Getting one's thoughts straight: a dialogical analysis of women's accounts of poorly controlled type 2 diabetes. Psychol Health. 2012;27(3):378-93.

87. Harvey JN, Lawson VL. The importance of health belief models in determining self-care behaviour in diabetes. Diabet Med. 2009;26(1):5-13.

88. Hawkins A, Look R. Levels of engagement and barriers to physical activity in a population of adults with learning disabilities. Br J Learn Disabil. 2006; 34(4):220-6

89. Majeed-Ariss R, Jackson C, Knapp P, Cheater FM. British-Pakistani women's perspectives of diabetes self-management: the role of identity. J Clin Nurs. 2015;24(17-18):2571-80.

90. Nagelkerk J, Reick K, Meengs L. Perceived barriers and effective strategies to diabetes self-management. J Adv Nurs. 2006;54(2):151-8.

91. Nam S, Chesla C, Stotts NA, Kroon L, Janson SL. Barriers to diabetes management: patient and provider factors. Diabetes Res Clin Pract. 2011; 93(1):1-9.

92. Nath C. Literacy and diabetes self-management. Am J Nurs. 2007;107(6):43-9.

93. Perri MG, Sears SF, Clark JE. Strategies for improving maintenance of weight loss: toward a continuous care model of obesity management. Diabetes Care. 1993;16(1):200-9.

94. Rouse L, Finlay WM. Repertoires of responsibility for diabetes management by adults with intellectual disabilities and those who support them. Sociol Health Illness. 2016;38(8):1243-57.

95. Skovlund SE, Peyrot M. The Diabetes Attitudes, Wishes, and Needs (DAWN) program: a new approach to improving outcomes of diabetes care. Diabetes Spectrum. 2005;18(3):136-42
96. Trief P, Sandberg JG, Ploutz-Snyder R, Brittain R, Cibula D, Scales K, Weinstock RS. Promoting couples collaboration in type 2 diabetes: the diabetes support project pilot data. Fam Syst Health. 2011;29(3):253.

97. Whitehead LC, Trip HT, Hale LA, Conder J. Negotiated autonomy in diabetes self-management: the experiences of adults with intellectual disability and their support workers. J Intellect Disabil Res. 2016;60(4):389-97.

98. Winkley K, Evwierhoma C, Amiel S, Lempp H, Ismail K, Forbes A. Patient explanations for non-attendance at structured diabetes education sessions for newly diagnosed type 2 diabetes: a qualitative study. Diabet Med. 2015; 32(1):120-8.

99. Zulman DM, Rosland AM, Choi H, Langa KM, Heisler M. The influence of diabetes psychosocial attributes and self-management practices on change in diabetes status. Patient Educ Couns. 2012;87(1):74-80.

100. Deakin TA, Cade JE, Williams R, Greenwood DC. Structured patient education: the diabetes X-PERT Programme makes a difference. Diabet Med 2006;23(9):944-54.

101. Funnell MM, Anderson RM. Empowerment and self-Management of Diabetes. Clin Diabetes. 2004;22(3):123-7.

102. Group DPPR. The diabetes prevention program (DPP). Diabetes Care. 2002; 25(12):2165-71.

103. Skinner TC, Carey ME, Cradock S, Daly H, Davies MJ, Doherty Y, Heller S, Khunti K, Oliver L. Diabetes education and self-management for ongoing and newly diagnosed (DESMOND): process modelling of pilot study. Patient Educ Couns. 2006;64(1):369-77.

104. Carroll C, Booth A, Cooper K. A worked example of" best fit" framework synthesis: a systematic review of views concerning the taking of some potential chemopreventive agents. BMC Med Res Methodol. 2011;11(1):29.

105. Mingers J, Rosenhead J. Problem structuring methods in action. Eur J Oper Res. 2004;152(3):530-54.

106. Graham L, Wright J, Walwyn R, Russell AM, Bryant L, Farrin A, House A. Measurement of adherence in a randomised controlled trial of a complex intervention: supported self-management for adults with learning disability and type 2 diabetes. BMC Med Res Methodol. 2016;16(1):132

107. Jahoda A, Melville C, Cooper S-A, Hastings R, Briggs A, Dagnan D, Hatton C, McConnachie A, Williams C, Jones RP. BEAT-IT: a randomised controlled trial comparing a behavioural activation treatment for depression in adults with intellectual disabilities with an attention control: summary of study protocol. Trials. 2015;16

108. Jahoda A, Melville C, Pert C, Cooper SA, Lynn H, Williams C, Davidson C. A feasibility study of behavioural activation for depressive symptoms in adults with intellectual disabilities. J Intellect Disabil Res. 2015;59(11):1010-21.

109. Greaves CJ, Wingham J, Deighan C, Doherty P, Elliott J, Armitage W, Clark M, Austin J, Abraham C, Frost J. Optimising self-care support for people with heart failure and their caregivers: development of the Rehabilitation Enablement in Chronic Heart Failure (REACH-HF) intervention using intervention mapping. Pilot and feasibility studies. 2016;2(1):37.

110. Taggart L, Coates V, Clarke M, Bunting B, Davies M, Carey M, Northway R, Brown M, Truesdale-Kennedy M, Martin-Stacey L. A study protocol for a pilot randomised trial of a structured education programme for the selfmanagement of type 2 diabetes for adults with intellectual disabilities. Trials. 2015;16(1):148

\section{Ready to submit your research? Choose BMC and benefit from:}

- fast, convenient online submission

- thorough peer review by experienced researchers in your field

- rapid publication on acceptance

- support for research data, including large and complex data types

- gold Open Access which fosters wider collaboration and increased citations

- maximum visibility for your research: over $100 \mathrm{M}$ website views per year

At BMC, research is always in progress.

Learn more biomedcentral.com/submissions 